\title{
Die Quartärforschung an den deutschen Hochschulen
}

\author{
Von Paul Woldstedt, Bonn
}

In der Quartärforschung hat die deutsche Wissenschaft von jeher eine bedeutende Rolle gespielt. Man denke nur an Namen wie Albrecht Penck, Felix Wahnschaffe, Konrad KeIlHack, Wolfgang Soergel und viele andere. Das ist nicht Zufall, sondern hat seinen Grund darin, daß am deutschen Boden das Quartär einen ganz beträchtlichen Anteil hat. Nicht nur das ganze Norddeutsche Flachland ist aus quartären Bildungen zusammengesetzt, sondern ebenso ein großer Teil des Alpenvorlandes. Ja, auch im dazwischen gelegenen Gebiet spielen quartäre Sedimente wie Löß, Flußschotter usw. eine wichtige Rolle.

So sehr also Bildungen der Quartärformation am deutschen Boden beteiligt sind, so wenig wird im allgemeinen hierauf im Lehrbetrieb der deutschen Hochschulen Rücksicht genommen. Wohl wird gelegentlich eine Spezialvorlesung über das Eiszeitalter von geologischer oder geographischer Seite gehalten. Aber in der eigentlichen A usbildung der Geologen spielt das Quartär eine sehr geringe Rolle. Das ist ein Zustand, der dringend der Änderung bedarf - schon allein aus praktischen Gründen. Wieviel Geologen werden an Geologischen Landesämtern angestellt und in Gebieten mit quartären Schichten eingesetzt, obwohl sie zunächst nicht über genügende Kenntnisse im Quartär verfügen. Sie haben vor allem nicht die notwendige Erfahrung in den verschiedenen Methoden, die heute für eine exakte Erforschung des Quartärs unerläßlich sind. Diese Kenntnisse und Erfahrungen, die sie eigentlich mitbringen sollten, müssen sie sich - das habe ich oft genug an den Geologischen Landesämtern, an denen ich tätig war, erlebt - mühsam in den ersten Jahren ihrer Berufstätigkeit aneignen.

Hydrogeologische Gutachten, Baugrundgutachten. und dgl. spielen heute in der Tätigkeit der Ämter eine ganz überragende Rolle. Sie setzen nicht nur im Flachlande, sondern vielfach auch in anderen Gebieten eine genaue Kenntnis gerade der quartären Ablagerungen, vor allem ihrer räumlichen Erstreckung, voraus. Daß für alle Fragen der Bodenkunde, der Land- und Forstwirtschaft usw., das Quartär eine überragende Rolle spielt, das bedarf keiner besonderen Hervorhebung.

Ja, selbst mancher Erdölgeologe, der seine Bohrungen im Flachland ansetzen muß, hat schon darüber geklagt, daß er leider auf der Universität viel zu wenig über das Quartär gelernt habe. -

Unter den deutschen Hochschulen gab es immer einige, in denen das Quartär mehr oder weniger gepflegt wurde. Lange Zeit war Kiel eine Stätte, an der das der Fall war (HAAS, Wüst, GripP).

Auch in Rostock (E. Geinitz), Berlin und Hamburg (C. GotTsche) spielte das Quartär eine gewisse Rolle. In Greifswald begann vor dem letzten Kriege K. Richter die Quartärforschung auszubauen. Infolge des Krieges und der Nachkriegsentwicklung war leider eine Fortsetzung dieser Bestrebungen nicht möglich. Auch dort, wo W. Soergex wirkte, d. h. zunächst in Breslau, später in Freiburg i. Br., entstand etwas wie systematische Quartärforschung, die wenigstens in Freiburg auch weitergeführt wurde.

In anderen Ländern (Verein. Staaten, Nordische Länder, Niederlande usw.) ist die Notwendigkeit, das Quartär in stärkerem Maße bei der Ausbildung der Geologen zu berücksichtigen, eher erkannt worden. In Schweden z. B. existiert an jeder der drei Hauptuniversitäten innerhalb der Geologisch-Mineralogischen Institute eine besondere quartärgeologische Abteilung, die mit einem Dozenten im Range eines Extraordinarius besetzt ist. Dabei könnte man schwerlich behaupten, daß das Quartär in Skandinavien 
eine größere Rolle spiele als etwa in Norddeutschland. Im Gegenteil, das Quartär umfaßt dort im wesentlichen nur die Spät- und Nacheiszeit und bildet lediglich eine lückenhafte Decke über dem älteren Gebirge. Trotzdem hat man es für notwendig erachtet, besondere Professuren für Quartärgeologie einzurichten. Abgesehen davon, war lange Zeit hindurch eine der wichtigsten nordischen Geologie-Professuren - die in Stockholm - mit Quartärgeologen besetzt (Gerard DE GEer, Lennart von Post).

Es erscheint danach dringend erforderlich, daß das Quartär auch an den deutschen Universitäten eine wesentlich stärkere Pflege findet. Der Ordinarius für Geologie kann normalerweise die Quartärgeologie nicht nebenbei mitbetreiben. Sein Lehrgebiet ist so groß und so umfassend, daß er in den seltensten Fällen dazu kommen wird, das Quartär in seiner Hauptvorlesung über Erdgeschichte (Formationsgeschichte) überhaupt nur zu behandeln - ganz abgesehen davon, daß das Gebiet manchen Ordinarien recht fremd ist, ja einzelnen als unnötig und kaum zur „echten“ Geologie zugehörig erscheint. Und doch ist das Quartär eine echte geologische Formation, die wesentlich mit geologischen Methoden zu erforschen ist. Gewiß kommen noch andere Methoden dazu, wie etwa die geomorphologische, die in Deutschland vorwiegend von Geographen betrieben wird. Aber die Hauptmethode, die stratigraphische, ist eine geologische, und es kann keinem Zweifel unterliegen, daß die Erforschung der Quartärformation und ihre Lehre auf den Universitäten im wesentlichen in die Hand des Geologen gehört, wie das ja auch in allen maßgeblichen Ländern der Welt der Fall ist.

So muß die Forderung aufgestellt werden, daß wenigstens an einer Reihe von deutschen Hochschulen im Rahmen der geologischen Institute Lehrstellen errichtet werden, die der besonderen Pflege des Quartärs dienen. Vor allem im Norddeutschen Flachlande sind solche unbedingt erforderlich. Es ist doch eigentlich ein Unding gewesen, daß im Zentrum des Norddeutschen Flachlandes, in Berlin, niemals wenigstens eine außerordentliche Professur für Quartärgeologie bestanden hat. Gewiß, es waren Dozenten da, früher F. W AHNSCHAFFe, später F. Solger, die über das Quartär lasen. Aber sie hatten keine amtliche Stellung, und vor allem fehlte ihnen das Institut mit den notwendigen Laboratorien usw.

Neben den norddeutschen erscheinen aber auch einige süddeutsche Lehrstellen im Bereiche des voralpinen Quartärs notwendig. Schließlich ist es dringend erwünscht, daß auch an einzelnen mitteldeutschen Universitäten das Quartär stärker gepflegt wird. Lö1\}, Terrassen, Höhlenablagerungen, Säugetierfaunen usw. sind hier die wichtigsten Objekte, die einer stärkeren Pflege seitens der Geologen bedürfen. -

Der erste Einwand, der sofort erhoben werden wird, ist der: warum denn eine Sonderbehandlung des Quartärs? Können dann nicht mit demselben Rechte Professuren etwa für das Devon, den Jura, das Tertiär usw. gefordert werden? Abgesehen davon, daß solche $\mathrm{ja}$ in gewisser Weise bestehen, insofern als manche Universitäten auf Grund ihrer Lage und Tradition sich vorwiegend mit bestimmten Formationen befassen, ist aber doch ein grundlegender Unterschied vorhanden: es läßt sich nun einmal nicht leugnen, daß annähernd die Hälfte des deutschen Bodens von quartären Bildungen eingenommen wird, d. h. daß eben das Quartär die verbreitetste geologische Formation in Deutschland ist. Das gilt im übrigen nicht nur für Deutschland, sondern für die ganze Welt. Überall hat sich der Geologe mit dem Quartär auseinanderzusetzen, sei es auch nur durch die Morphologie und die Bodenbildung in Gebieten, wo sonst nur älteres Gebirge die Oberfläche bildet.

Hinzukommt, daß für die Erforschung des Quartärs zahlreiche spezielle Methoden erforderlich sind, die bei anderen Formationen keine oder jedenfalls eine geringere Rolle spielen. Nur die wichtigsten können hier angeführt werden.

Zunächst muß der Quartärgeologe eine ganz gründliche Ausbildung in der B od e n $\mathrm{k}$ u n d e erhalten. Nur die richtige Diagnose eines fossilen Bodens gestattet dem Quartär- 
geologen, Schlüsse zu ziehen auf das Klima oder die Bedingungen, unter denen sich der betreffende Boden gebildet hat. So ist z. B. eine exakte Lößgliederung im wesentlichen nur durch genaue bodenkundliche Untersuchung der eingeschalteten fossilen Böden möglich gewesen (vgl. z. B. die Untersuchungen von E. SснӧNHALs). Die nordamerikanischen Quartärgeologen sind in dieser Beziehung weiter als die deutschen. In ihren Arbeiten findet man durchweg einwandfreie Beschreibungen fossiler Böden, was man noch nicht von allen deutschen Arbeiten sagen kann.

Die Bodenkunde, die dem Quartärgeologen wichtige Hinweise gibt, ob ein fossiler Boden in einem warmen oder kalten, in einem feuchten oder trockenen Klima gebildet worden ist, ob es sich um eine interglaziale oder eine interstadiale Bildung handelt, muß ergänzt werden durch spezielle Kenntnisse in bestimmten Zweigen der Paläontologie, die von den übrigen Geologen weniger betrieben werden. In erster Linie gehört hierher die Pollena nalyse, deren Grundzüge der Quartärgeologe beherrschen muß. Er muß zum mindesten in der Lage sein, eine einfache pollenanalytische Untersuchung durchzuführen und entsprechend auszuwerten. Oft muß die Pollenanalyse noch ergänzt werden durch D i a tome en - und Ostrakoden-Analysen.

Weiter muß der Quartärgeologe eine besondere Ausbildung erhalten in einzelnen Teilen der Paläozoologie, die wiederum in der allgemeinen paläontologischen Ausbildung nur eine geringe Rolle spielen. Es handelt sich dabei einmal um die S ch n e ck en (und gewisse Gruppen von Muscheln) und zweitens um die Sä u g e t i e re. Gerade die quartären Säugetiere werden in den normalen Vorlesungen und Übungen der Paläontologie nur verhältnismäßig kurz behandelt, was jedenfalls für die Ausbildung des Quartärgeologen nicht genügt.

Ein weiteres spezielles Gebiet, in dem der Quartärgeologe eine besondere Ausbildung erhalten muß, ist der Bereich der modernen sedimentpet rographis chen Me tho d e n. Es ist hier nicht nur an schwermineralanalytische Untersuchungen gedacht, ohne die heute wichtige Fragen der Quartärgeologie nicht zu lösen sind (z. B. Terrassen-Untersuchungen u. dgl.), sondern auch an die moderne Geröll- und Geschiebeforschung, die sedimentpetrographische Untersuchung der Höhlenablagerungen, wie sie R. LAIS und Elisabeth SCHMID entwickelt haben, und zahlreiche andere.

Ebenso wichtig sind die modernen Methoden der Gefügeforschung, d. h. insbesondere z. B. Schrägschichtungsmessungen u. dgl. Sie sind für die Herkunft aller Arten von Aufschiittungen usw. besonders bedeutsam.

Schließlich muß der Quartärgeologe eine zusätzliche Ausbildung erhalten in der U r g e s ch i cht e. Die Haupttatsachen der Menschheitsentwicklung müssen ihm ebenso vertraut sein wie die wichtigsten Formen paläolithischer Artefakte. Bei allen seinen Untersuchungen im Gelände muß der Quartärgeologe darauf gefaßt sein, auf Spuren des vorgeschichtlichen Menschen zu stoßen. Er muß selber in der Lage sein, sie zu beurteilen, vor allem auch, wie weit sie etwa stratigraphisch wichtig sein können.

So ist es ein ganzer Komplex von Hilfswissenschaften, Methoden usw., die für die Ausbildung des normalen Geologen nur eine geringe Rolle spielen, die aber für den Quartärgeologen unerläßlich sind und die er bisher nur in den seltensten Fällen an der Universität lernt. -

Als zweiter Einwand wird mir dann entgegengehalten werden: solange wir nicht an jeder Universität neben dem Geologen einen Paläontologen haben, solange ist an besondere Stellen für Quartärgeologen gar nicht zu denken. Ich möchte demgegenüber betonen, daß mir das eine so notwendig erscheint wie das andere. Aber eine grundsätzliche Rangordnung scheint mir hier nicht am Platze zu sein. Solange man nicht an jeder Universität beides einrichten kann, schaffe man an der einen Stelle einen Lehrstuhl für Paläontologie, an der andern einen für Quartärgeologie. 
Schließlich mag die Frage aufgeworfen werden: stehen denn die nötigen Kräfte dafür zur Verfügung? Darauf muß geantwortet werden: ja, sie stehen zur Verfügung, und zwar für die verschiedenen Richtungen. Denn im einzelnen wird die Arbeitsrichtung der Quartärgeologen recht verschieden sein können. Bei dem einen werden die Beziehungen zur Bodenkunde besonders wichtig sein. Es wurde oben schon darauf hingewiesen, daß die richtige Beurteilung der fossilen Böden für die Stratigraphie des Quartärs von ganz besonderer Bedeutung ist. Hier ist noch ein weites Arbeitsfeld für den bodenkundlich voll ausgebildeten Quartärgeologen vorhanden. ${ }^{1}$ )

Daß andererseits im Norddeutschen Flachlande die Geschiebe- und Geröllforschung im weitesten Sinne immer eine der wichtigsten Arbeitsmethoden sein wird, braucht nicht besonders hervorgehoben zu werden.

Von anderen werden wiederum die Beziehungen zur Paläontologie stärker gepflegt werden, so insbesondere zur Säıgetierforschung, wie sie etwa W. SoEngEL betrieben hat. Heute sitzen solche Säugetierforscher vorwiegend an den Museen. Ihre stärkere Einschaltung in den Lehrbetrieb der Universitäten könnte nur von Vorteil sein. Dasselbe gilt für die Molluskenforscher, deren es z. Zt. an den deutschen Universitäten überhaupt nur ganz wenige gibt.

Was die Pollenanalyse anbelangt, so ist sie als wissenschaftliche Forschungsmethode von Botanikern und Geologen entwickelt und zunächst auf die Postglazialzeit, später auf die Interglaziale angewandt worden. Wenn ihr Anwendungsbereich heute auch über das Quartär hinausgeht, so hat der Pollenanalytiker doch zweifellos seinen wichtigsten Platz innerhalb der Quartärforschung.

Um schließlich zu den Beziehungen zur Urgeschichte zu kommen, so haben W. SoergeI. und seine Schüler gezeigt, was hier auf diesem wichtigen Grenzgebiet bei systematischer Forschung an neuen Erkenntnissen herausgebracht werden kann.

So besteht jedenfalls keine Gefahr, daß etwa der Umfang der Quartärgeologie zu klein für die Schaffung besonderer Professuren sei. -

Es soll nun nicht etwa der Auffassung das Wort geredet werden, daß an den Universitäten besondere Quartärgeologen heranzuzüchten wären, die von der übrigen Geologie nichts zu wissen brauchten. Davon kann natürlich keine Rede sein. E in b e s o n d e res Quartärstudium hätte a ufzubauen auf einer vollen geologis c he n A usbildung - genau wie das für einen Paläontologen gefordert wird. Wie für diesen ist es eine Spezialausbildung, die erst in späteren Semestern zu erfolgen hätte. Das wirkliche Erfassen der Stratigraphie - das Wesentlichste in der Geologie, - das muß dem Quartärgeologen wie dem Paläontologen vor ihrer Spezialausbildung in Fleisch und Blut übergegangen sein.

Auch trete ich nicht etwa dafür ein, daß nun besondere quartärgeologische Institute an einzelnen Universitäten zu errichten seien. Ich halte es für viel besser, daß so verfahren wird wie in Schweden, daß nämlich an bestehenden geologischen Instituten Abteilungen für Quartärgeologie eingerichtet werden. Die Aufteilung in besondere Institute ist in Deutschland in manchen Sparten schon reichlich weit gegangen. Es erfordert das in vielen Fällen nicht nur Mehr-Anschaffung von Apparaten, Büchern usw., sondern auch einen Mehraufwand von Verwaltungsarbeit, Hilfspersonal usw. Ja, oft wird in sachlich benachbarten Instituten über die gleichen Gebiete geforscht und gelehrt. So ist zweifellos die Einrichtung von Abteilungen in größeren Instituten vorzuziehen.

1) Es könnte hier die Frage aufgeworfen werden: ist es denn nicht richtiger, gleich einen vollen Bodenkundler statt eines Quartärgeologen einzustellen? Mir scheint der Platz des reinen Bodenkundlers in erster Linie an den land- und forstwissenschaftlichen Hochschulen oder Fakultäten zu sein, während an die geologischen Institute der Quartärgeologe (mit gründlicher bodenkundlicher Ausbildung) gehört. 
Etwas anderes wäre die Schaffung eines zentralen reinen Forschungsinstitutes für Quartärfragen im weitesten Sinne (einschließlich der Urgeschichte, der absoluten Chronologie mit Hilfe moderner Zeitbestimmungsmethoden usw.). Ein solches Institut, das den weltweiten Fragen der Quartärformation und insbesondere der Entwicklung der Menschheit und ihren Kulturen nachzugehen hätte, könnte die Quartärabteilungen in den geologischen Instituten der Universitäten in wertvoller Weise ergänzen.

Manuskr. eing. 15. 12. 1958.

Anschrift des Verf.: Prof. Dr. Paul Woldstedt, Bonn, Argelanderstraße 118.

Nachtrag während des Druckes. Aus einem besonderen Anlaß muß noch auf Folgendes hingewiesen werden. Es steht fest, daß in Deutschland z. Zt. viel zu wenig iiber das Quartär gearbeitet wird. An den Hochschulen geschieht dies nicht, weil keine Professuren für Quartärgeologie und infolgedessen auch nur wenig Doktoranden für dies Gebiet vorhanden sind. Die Geologischen Landesämter sind mit praktischen Aufgaben so überlastet, daß ihre Mitglieder nur selten noch zu größeren wissenschaftlichen Arbeiten kommen, vor allem nicht im Quartär. So ergibt sich der gegenwärtige Zustand, daß auf wichtigen Teilgebieten des deutschen Quartärs ausländische Forschungen immer mehr zunehmen. Gewiß will man den Ausländern nicht verbieten, in Deutschland zu arbeiten. Aber eigentlich sollte es doch in erster Linie Aufgabe deutscher Forscher sein, diese Untersuchungen im eigenen Lande auszuführen.

So ergibt sich auch hieraus die dringende Notwendigkeit, der Quartärforschung an den deutschen Hochschulen einen größeren Raum zuzuerkennen. 